\title{
Numerical Simulation on the Penetration Behavior of the Projectile during the Water Injection Stage of Water-Projectile-Assisted Injection Molding Process
}

\author{
Tangqing Kuang $\mathbb{D}^{1},{ }^{1}$ Qiang Feng $\mathbb{D}^{1},{ }^{1}$ Tian Liu, ${ }^{1}$ Luohao Zhong, ${ }^{1}$ Yanqing Wang, \\ and Hesheng Liu (i) ${ }^{2}$ \\ ${ }^{1}$ East China Jiaotong University, Nanchang 330013, Jiangxi, China \\ ${ }^{2}$ East China University of Technology, Nanchang 330013, Jiangxi, China \\ Correspondence should be addressed to Tangqing Kuang; 545960963@qq.com
}

Received 22 May 2019; Revised 25 July 2019; Accepted 4 September 2019; Published 28 March 2020

Guest Editor: Yun Zhang

Copyright (c) 2020 Tangqing Kuang et al. This is an open access article distributed under the Creative Commons Attribution License, which permits unrestricted use, distribution, and reproduction in any medium, provided the original work is properly cited.

\begin{abstract}
Water-projectile-assisted injection molding (W-PAIM) is a novel molding process for plastic pipes with complicated shape. It utilizes high-pressure water as a power to push a solid projectile to penetrate through the melt to form a hollow space. In order to investigate the penetration behavior of the projectile during the water injection stage of W-PAIM process, numerical simulation of the water injection stage of a W-PAIM pipe with straight and curved segments was carried out. A turbulent flow for the driving water was considered in the motion equation, and the dynamic mesh technology was used to deal with the moving solid projectile. The simulation results, including RWT and the flow fields, were compared with those of water-assisted injection molding (WAIM) pipe with the same outer dimensions. It was found that the residual wall thickness (RWT) of the W-PAIM pipe is much thinner than that of the WAIM pipe. The projectile has a crucial influence on the RWT. The pressure fields of W-PAIM and WAIM are very similar in both straight and curved segments. The velocity field and strain rate field near the penetration front in W-PAIM are quite different from those in WAIM due to the drag flow caused by the projectile penetration.
\end{abstract}

\section{Introduction}

The processing of plastic products occupies a large proportion in the current manufacture industry. [1] Waterassisted injection molding (WAIM) is one of the innovations of plastic injection molding processes [1]. In the Institute of Plastics Processing in Germany [2, 3], WAIM is developed to produce hollow or partially hollow parts in 1998. In the process, pressurized water following the melt injection step is injected into the core of the melt to form a hollow product. The development of this molding technique was made as a variant of the well-known gasassisted injection molding process (GAIM), in which gas is used to core out the product. Both GAIM and WAIM techniques might produce complex pipe products with thin and thick sections, which are present in various branches of industry. Simultaneously, compared to the conventional injection molding process, they have the main strength of improving product characteristics and lowering production costs on the hollow products partiality. The use of water instead of gas leads to a further enhancement of some product characteristics and reduction of process costs, which is principally associated with the physical properties of water. More details on the specific advantages and disadvantages of both techniques are already extensively documented in earlier studies $[4,5]$.

Fluid-projectile-assisted injection molding (FPAIM, PIT in German) process, which is one process variant of fluidassisted injection molding (FAIM, including WAIM and GAIM), can overcome the limitations of FAIM such as thicker residual wall thickness (RWT), fluctuation of RWT, and material selection. It was first documented in a Japanese patent [6]. In the process, a hollow channel in a part is 
formed by a projectile which is pushed to core out polymer melt by a pressurized fluid. Compared with GAIM or WAIM, thinner and more uniform wall thicknesses with smoother surfaces can be achieved [7]. Moreover, the FPAIM process possesses a wider range of material applicability because molten core is displaced by a rigid projectile and the fluid comes only after the RWT is formed. FPAIM has two methods: short shot method and full shot method. The main difference between them is whether the cavity is partially or fully filled with a resin melt before the water injection. Analogous to the FAIM process, the FPAIM process can be classified as water-projectile-assisted injection molding (W-PAIM) and gas-projectile-assisted injection molding (G-PAIM). Liquid water has a higher cooling efficiency and can shorten cycle times in the W-PAIM process. Many researchers have investigated the penetration behaviors of water, the distribution of the RWT [8-12], and the optimization of processing parameters in WAIM via experimentation [13-15]. However, to the best knowledge of the authors, only some researchers from the Institute of Plastic Processing (IKV) at RWTH Aachen University have studied the FPAIM process via experimentation. They showed the potential and limitations of the new process [16-18]. As far as the authors know, the penetration behavior of the projectile during the water injection stage of W-PAIM process has not been theoretically studied.

This present research is devoted to studying the characteristics of flow filed and the penetration behavior of the projectile during the water injection stage of W-PAIM process. A W-PAIM pipe with straight and curved segments was used as a test case, and numerical simulation of the water injection stage of it was conducted. This study includes the following: (1) the influence of projectile on the size of penetrating section and the distribution of RWT; (2) the characteristics of velocity field, pressure field, and strain rate field when the projectile is penetrating through the straight segment; and (3) the flow field when the projectile is penetrating through the curved segment. The research results are helpful to understand the penetration mechanism of the projectile in melt and provide a theoretical basis for the process control of W-PAIM and quality improvement of W-PAIM part.

\section{Modeling and Method}

2.1. Mathematical Model. Generalized Hele-Shaw model was widely used in the numerical simulations of the conventional injection molding. But it is limited to the thin-walled cavity and laminal flow. The water injection stage of W-PAIM is a multiphase stratified unsteady flow involving fluid-structure interaction, which includes the non-Newtonian laminar of the melt with low Reynolds number, the turbulence of the water injection with high Reynolds number, and the interaction among the melt, water, and the projectile. Therefore, it is necessary to improve the mathematical model. To simplify the model and facilitate the simulation, the simplification assumptions are as follows based on CFD method: the water and melt are incompressible; the melt flow meets no-slip boundary; the body forces and surface tension are ignored; the melt fills mold cavity uniformly at the initial state, and the melt injection process does not affect the projectile penetration; and the plastic projectile with a high melting point was regarded as a moving solid boundary without thermal properties.

Governing equations include the continuity equation, motion equation, energy equation, constitutive equation, viscosity model, and volume fraction equation.

Based on the simplifications that water and melt are incompressible, the continuity equation is given by

$$
\frac{\mathrm{d} u_{i}}{\mathrm{~d} x_{i}}=0
$$

where $u_{i}$ is the fluid velocity.

During the penetration of projectile, the driving water is high Reynolds number turbulence. Therefore, the random nature of turbulent flow must be considered in motion equations. The Reynolds time-averaged motion equation is given by

$$
\begin{aligned}
\frac{\partial}{\partial t}\left(\rho u_{i}\right)+\frac{\partial}{\partial x_{j}}\left(\rho u_{i} u_{j}\right)= & \frac{\partial}{\partial x_{j}}\left[\mu\left(\frac{\partial u_{i}}{\partial x_{j}}+\frac{\partial u_{j}}{\partial x_{i}}-\frac{2}{3} \delta_{i j} \frac{\partial u_{l}}{\partial x_{l}}\right)\right] \\
& -\frac{\partial p}{\partial x_{i}}+\frac{\partial}{\partial x_{j}}\left(-\rho \overline{u_{i}^{\prime} u_{j}^{\prime}}\right) .
\end{aligned}
$$

The equations are not closed and cannot be solved for the introduction of Reynolds stress in the last item of equation (2). To deal with Reynolds stress and make the equations close, the relationship between Reynolds stress and average velocity gradient is established based on the Bossiness eddy viscosity assumption:

$$
-\rho \overline{u_{i}^{\prime} u_{j}^{\prime}}=\mu_{t}\left(\frac{\partial u_{i}}{\partial x_{j}}+\frac{\partial u_{j}}{\partial x_{i}}\right)-\frac{2}{3}\left(\rho k+\mu_{t} \frac{\partial u_{i}}{\partial x_{i}}\right) \delta_{i j},
$$

where $\mu_{t}$ is the turbulent viscosity and $k$ is the turbulent kinetic energy.

In order to calculate the turbulent viscosity $\mu_{t}$, a standard $k-\omega$ turbulent model was adopted, in which $k$ is the turbulence kinetic energy and $\omega$ is the specific dissipation rate. The turbulent viscosity, $\mu_{t}$, is computed by combining $k$ and $\omega$ as follows:

$$
\mu_{t}=\alpha^{*} \frac{\rho k}{\omega}
$$

where $\alpha^{*}$ is the modified coefficient of low Reynolds number.

$k$ and $\omega$ are two basic unknown variables that are obtained from the following transport equations:

$$
\begin{gathered}
\frac{\partial}{\partial t}(\rho k)+\frac{\partial}{\partial x_{i}}\left(\rho k u_{i}\right)=\frac{\partial}{\partial x_{j}}\left[\left(\mu+\frac{\mu_{t}}{\sigma_{k}}\right) \frac{\partial k}{\partial x_{j}}\right]+G_{k}-Y_{k}, \\
\frac{\partial}{\partial t}(\rho \omega)+\frac{\partial}{\partial x_{i}}\left(\rho \omega u_{i}\right)=\frac{\partial}{\partial x_{j}}\left[\left(\mu+\frac{\mu_{t}}{\sigma_{k}}\right) \frac{\partial \omega}{\partial x_{j}}\right]+G_{\omega}-Y_{\omega} .
\end{gathered}
$$


The cooling of the polymer melt by the water and mold wall is considered during the penetration of projectile, and the energy equation is given by

$$
\frac{\partial}{\partial t}(\rho E)+\frac{\partial}{\partial x_{i}}\left(u_{i}(\rho E+p)\right)=\frac{\partial}{\partial x_{i}}\left(\lambda_{\mathrm{eff}} \frac{\partial T}{\partial x_{i}}+u_{i}\left(\tau_{i j}\right)_{\mathrm{eff}}\right),
$$

where $E$ denotes the total energy of the fluid, $\lambda_{\text {eff }}$ denotes the effective heat transfer coefficient, and $\left(\tau_{i j}\right)_{\text {eff }}$ denotes the deviatoric stress tensor.

The melt in W-PAIM is regarded as a generalized Newtonian fluid because it is mainly affected by shear stress. Therefore, the elastic behavior of melt is ignored, and the melt constitutive equations is given by

$$
\left(\tau_{i j}\right)_{\mathrm{eff}}=\eta(p, T, \dot{\gamma}) \dot{\gamma}
$$

where $T$ is temperature and $\dot{\gamma}$ is deformed velocity tensor.

Due to the direct cooling of melt by water, Cross-WLF viscosity model with seven parameters was adopted to characterize the viscosity of the melt in a wide temperature range. The expression of Cross-WLF viscosity model with seven parameters is as follows:

$$
\begin{aligned}
\eta(\dot{\gamma}, T, p) & =\frac{\eta_{0}(T, p)}{1+\left(\eta_{0} \dot{\gamma} / \tau^{*}\right)^{1-n}}, \\
\eta_{0}(T, p) & =D_{1} \exp \left(-\frac{A_{1}\left(T-T^{*}\right)}{A_{2}+\left(T-T^{*}\right)}\right), \\
T^{*}(p) & =D_{2}+D_{3} p, \\
A_{2} & =\bar{A}_{2}+D_{3} p,
\end{aligned}
$$

where $\eta_{0}$ is the zero shear viscosity; $\tau^{*}$ is the material constant related to shear stress level transited from Newton viscosity to power-law viscosity; $n$ is the non-Newtonian index in the high shear rate regime; and $T^{*}$ is the glass transition temperature. $A_{1}, \bar{A}_{2}, D_{1}, D_{2}$, and $D_{3}$ are material constants.

In order to make a comparison between the WAIM and $\mathrm{W}$-PAIM, polypropylene (PP) is selected as the molding polymer for its good molding properties of WAIM. The parameters of Cross-WLF model of PP are obtained from Moldflow material database and shown in Table 1.

As the water and melt are free surface flows without mutual penetration between the two phases, the volume-of-fluid (VOF) method can be used to track the free interface of water/melt twophase flow. The continuity equation of volume fraction is given by

$$
\frac{\partial \alpha_{i}}{\partial t}+u_{i} \frac{\partial \alpha_{i}}{\partial x_{i}}=0
$$

where $\alpha_{i}$ is the volume fraction of the $i$ th phase, which varies between 0 and 1. $\alpha_{i}$ takes the value greater than 0 and less than 1 which is referred to as the $i$ phase front.

2.2. Test Case Model and Boundary Conditions. A pipe with straight and curved segments was modeled as a test case to investigate the penetration behavior of the projectile during
TABLE 1: Cross-WLF viscosity model parameters of PP.

\begin{tabular}{lcccccc}
\hline$n$ & $\tau^{*}(\mathrm{~Pa})$ & $T_{g}(\mathrm{~K})$ & $D_{1}(\mathrm{~Pa} \cdot \mathrm{s})$ & $A_{1}$ & $A_{2}$ & $D_{3}$ \\
\hline 0.3083 & 16834.4 & 263.15 & $6.7266 \times 10^{13}$ & 30.441 & 51.6 & 0 \\
\hline
\end{tabular}

the water injection stage of W-PAIM process. It has a sectional diameter of $16 \mathrm{~mm}$, a deflection angle of $60^{\circ}$, and a bending radius of $20 \mathrm{~mm}$, as shown in Figure 1. A hollow projectile with a cylinder and a rounded head was used, as shown in Figure 2.

In order to solve the mathematical model, the boundary and initial conditions must be specified. Boundary conditions include the inlet, outlet, and wall boundaries, as shown in Figure 1 and specified as follows.

The inlet is a circle with a diameter of $6 \mathrm{~mm}$. Pressure inlet boundary condition is specified and the pressure at the inlet is water injection pressure. The pressure at the outlet is specified as atmospheric pressure. The temperature of inlet equals to water injection temperature:

$$
\begin{aligned}
p_{\text {inlet }} & =p_{\text {water }}, \\
p_{\text {outlet }} & =0, \\
T_{\text {inlet }} & =T_{\text {water }} .
\end{aligned}
$$

The wall meets no-slip boundary condition, and the fixed temperature boundary condition is specified, that is

$$
\begin{aligned}
& U_{\text {wall }}=0, \\
& T_{\text {wall }}=T_{\text {mold }} .
\end{aligned}
$$

The boundary condition of the moving projectile is specified as wall without thermal properties.

The molding process of simulation is full shot method of W-PAIM, which means the mold cavity is completely filled with melt before the water injection. Therefore, the whole flow field can be initialized as follows: the volume fraction of melt is 1 , the velocity of the melt is 0 , and the temperature of the melt is the melt injection temperature.

The values of processing parameters are shown in Table 2 .

The physical properties of PP and water shown in Table 3 are treated to be constant.

2.3. Numerical Procedure. Gambit software was applied for modeling and meshing of the test model. The numerical simulations were performed on FLUENT 16.0, a very famous fluid field simulation platform. A user-defined function was coded to calculate the melt viscosity.

In order to deal with the motion of rigid projectile, dynamic mesh in six degrees of freedom model, spring smoothing method, and local remeshing method were applied in the simulation.

The VOF model was adopted to track the free interface of water/melt two-phase flow. The pressure-implicit with splitting of operators (PISO) algorithm was used to solve the pressure-velocity coupling equation for its high accuracy and capability to deal with unsteady flow. The volume fraction discrete item is "QUICK," the pressure discrete item 


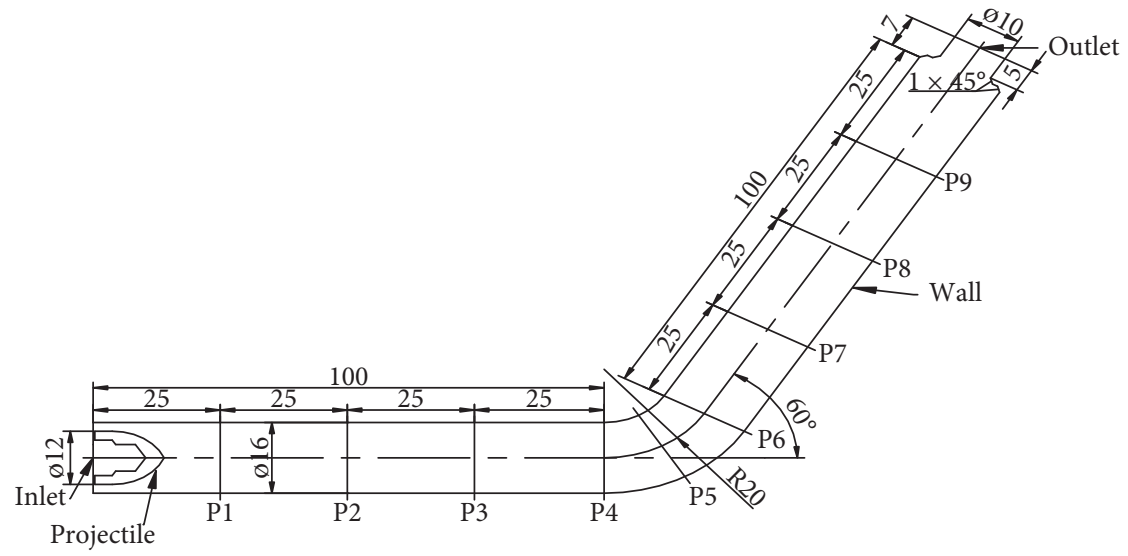

FIGURE 1: CFD model of a pipe with straight and curved segments for the simulations. All dimensions are in millimeters (no projectile for WAIM process).

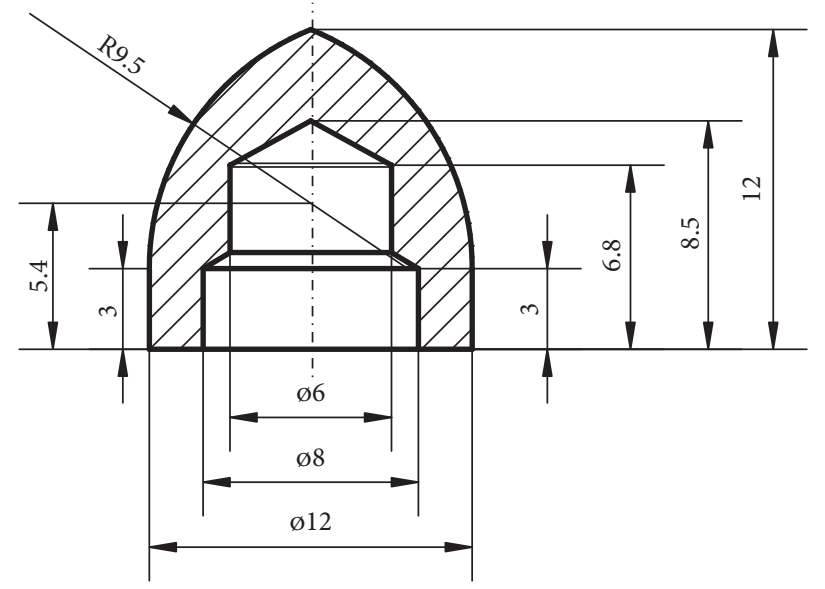

FIGURE 2: Projectile used in the simulation of W-PAIM process (All dimensions are in millimeters).

TABle 2: Processing parameters in the simulation.

Melt temp (K) Water pressure (MPa) Melt injection pressure (MPa) Holding time (s) Water delay time (s) Mold temp (K)

$\begin{array}{llllll}483 & 5 & 6 & 5 & 0 & 300\end{array}$

TABLE 3: Physical properties of melt and water in the simulation.

\begin{tabular}{lcccccc}
\hline Material & Density $\left(\mathrm{kg} \cdot \mathrm{m}^{-3}\right)$ & $\mathrm{Cp}\left(\mathrm{J} \cdot \mathrm{kg}^{-1} \cdot \mathrm{K}^{-1}\right)$ & $\begin{array}{c}\text { Thermal conductivity } \\
\left(\mathrm{W} \cdot \mathrm{m}^{-1} \cdot \mathrm{K}^{-1}\right)\end{array}$ & $\begin{array}{c}\text { Viscosity } \\
\left(\mathrm{kg} \cdot \mathrm{m}^{-1} \cdot \mathrm{s}^{-1}\right)\end{array}$ & $\begin{array}{c}\text { Standard state enthalpy } \\
\left(\mathrm{J} \cdot \mathrm{kg}^{-1} \cdot \mathrm{mol}^{-1}\right)\end{array}$ & $\begin{array}{c}\text { Reference } \\
\text { temperature }\end{array}$ \\
\hline PP & 775 & 3450 & 0.1875 & & 387940 & 483 \\
Water & 998.2 & 4182 & 0.6 & 1.003 & $-2.858 \times 10^{8}$ & 298 \\
\hline
\end{tabular}

is "PRESTO!", and the other items are "First-Order Upwind". For convergence and smoothness of solutions, the underrelaxation factors are all set to 0.3 and the time step size is fixed as $1 \mathrm{E}-5 \mathrm{~s}$.

\section{Results and Discussion}

3.1. RWT. Figures 3(a) and 3(b)show the penetration interface of WAIM and W-PAIM, respectively. As can be seen from that, both penetrations of water and projectile are successfully completed. Figure 3(c) shows the quantitative comparison of the RWTs at the specified positions between them. At the straight segments, the RWT of the WAIM pipe is about $2.1 \mathrm{~mm}$, while that of the W-PAIM pipe is about $1.4 \mathrm{~mm}$. At the curved segments, the RWTs of the WAIM pipe are $1.2 \mathrm{~mm}$ at the inner concave side and $2.8 \mathrm{~mm}$ at the outer convex side, while the RWTs of the W-PAIM pipe are $0.8 \mathrm{~mm}$ at the inner concave side and $2.0 \mathrm{~mm}$ at the outer convex side. The RWTs of the W-PAIM pipe are much thinner than those of the WAIM pipe. In 

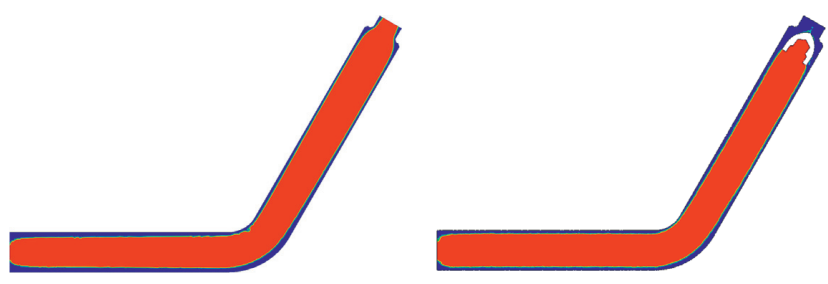

(a)

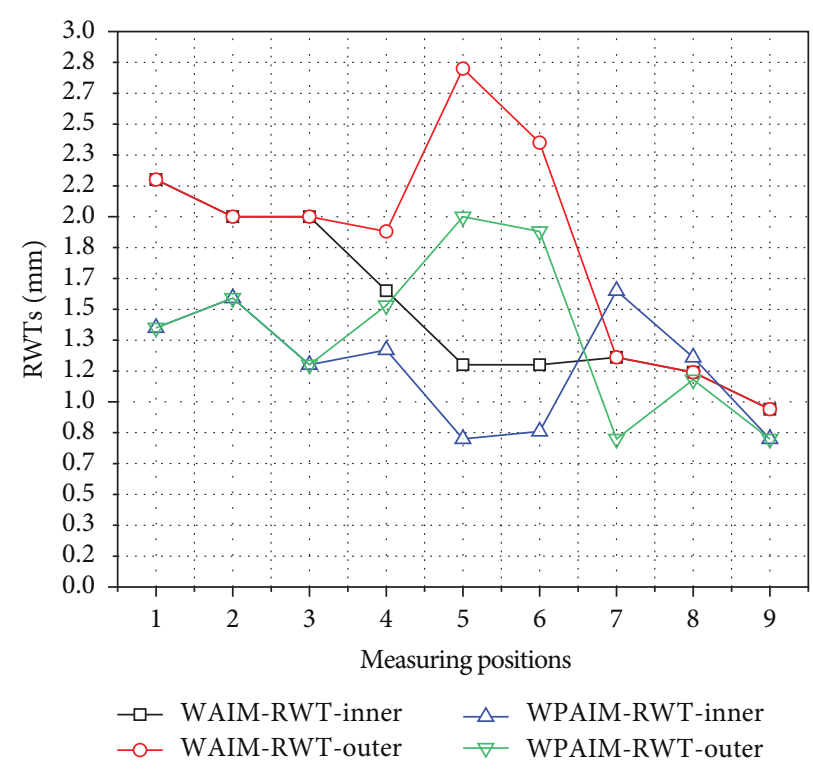

(c)

FIGURE 3: Comparison of penetration interfaces and RWTs between WAIM and W-PAIM pipe: (a) penetration interface of WAIM pipe, (b) penetration interface of W-PAIM pipe, and (c) RWTs.

addition, at the curved segments, the RWTs are thinner at the inner concave side and thicker at the outer convex side. This means that the penetrations of water in WAIM and the projectile in W-PAIM are relatively stable at the straight segment and always close to the inner concave side. The simulation results are in good agreement with the experimental results obtained by Hopmann and Behmenburg [17] and our preliminary work [19]. Obviously, the projectile has a crucial influence on the RWT. Moreover, it can be seen for both pipes that the RWTs at the second straight segments near the outlet show a decreasing trend along the flow direction. This is because in the simulation the outlet boundary is set to atmospheric pressure and the inlet pressure is water injection pressure. As the penetration proceeds, the pressure gradient in the melt in front of the penetration front increases gradually, and the penetration cross section increases correspondingly. The uniform penetration cross section can be obtained by designing a slender rather than a bulky overflow cavity.

In order to investigate the effects of different projectile diameters on RWT, different diameters projectile designs, such as $0 \mathrm{~mm}(0 \mathrm{~mm}$ represents the water-assisted injection molding experiment without a projectile), $6 \mathrm{~mm}, 8 \mathrm{~mm}$, $10 \mathrm{~mm}$, and $12 \mathrm{~mm}$, were explored and compared with WAIM.

As shown in Figure 4, the RWT measurement scheme is that six pipe samples are taken for each group experiment and each pipe takes four-section positions (P1, P2, P3, and P4) along the flow direction. Each section takes four equal points (P5, P6, P7, and P8) to measure wall thickness. All wall thickness data were recorded and the arithmetic mean was used to acquire the average RWT. The expression illustrating the arithmetic mean method for solving the RWT is as follows:

$$
\begin{aligned}
t_{P 1} & =\frac{t_{P 5}+t_{P 6}+t_{P 7}+t_{P 8}}{4}, \\
T_{P 1} & =\frac{t_{P 1}+t_{P 2}+t_{P 3}+t_{P 4}}{4}, \\
A_{0} & =\frac{T_{P 1}+T_{P 2}+T_{P 3}+T_{P 4}+T_{P 5}+T_{P 6}}{4},
\end{aligned}
$$

where $t_{P i}$ is the average RWT at $i$ th transverse section position at the same pipe, $T_{P i}$ is the average RWT at $i$ th pipe, and $A_{i}$ is the average RWT at $i$ th type of pipe.

Figure 5 shows the comparison of the cross section of test samples molding by different projectile diameters, including transverse section (Figure 5(a)) and longitudinal section (Figure 5(b)). It might be seen that the RWT of the pipe shaped with a cross-sectional diameter of $6 \mathrm{~mm}$ is large. The wall thickness distribution of the pipe is nonuniform. When the diameter of the projectile is less than $10 \mathrm{~mm}$, there is a certain deflection phenomenon during projectile passing the melt, which makes the wall thickness distribution of the pipe uneven. Therefore, the RWT of the pipe is mainly affected by the extrusion of the projectile and the water pushing the melt. At the same time, to obtain the average RWT, enough measuring data points of each test sample will be taken. More samples are measured. As shown in Figure 5(c), it can be found that when the diameter of the projectile is $6 \mathrm{~mm}$, the RWT of the pipe is $2.78 \mathrm{~mm}$. When the diameter of the projectile is $8 \mathrm{~mm}$, the RWT of the pipe is $2.39 \mathrm{~mm}$. According to the result of the error bar, the figure shows that when the diameter of the projectile is less than $10 \mathrm{~mm}$, the RWT fluctuates greatly. However, when the diameter of the projectile is larger than $10 \mathrm{~mm}$, the RWT of the pipes is mainly affected by the size of the projectile. The RWT is thinner than the WAIM process. According to the trend of 


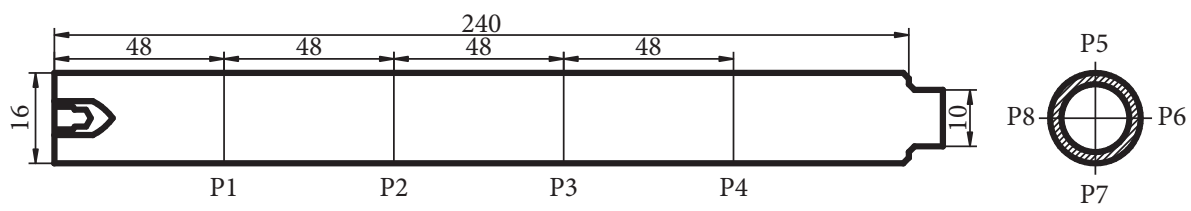

FIGURE 4: RWT measurement scheme.

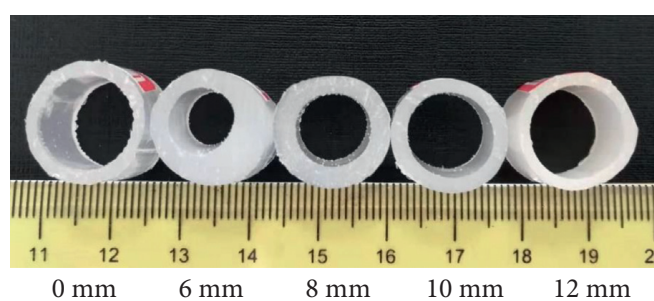

(a)

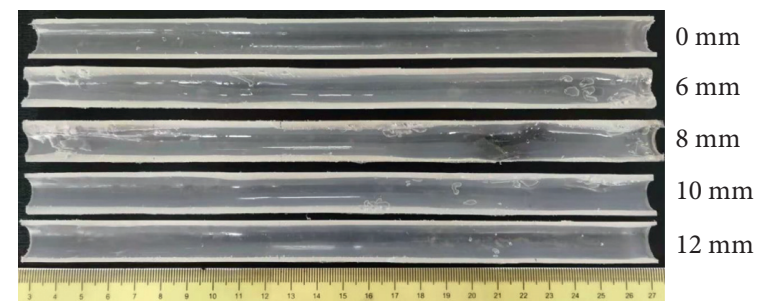

(b)

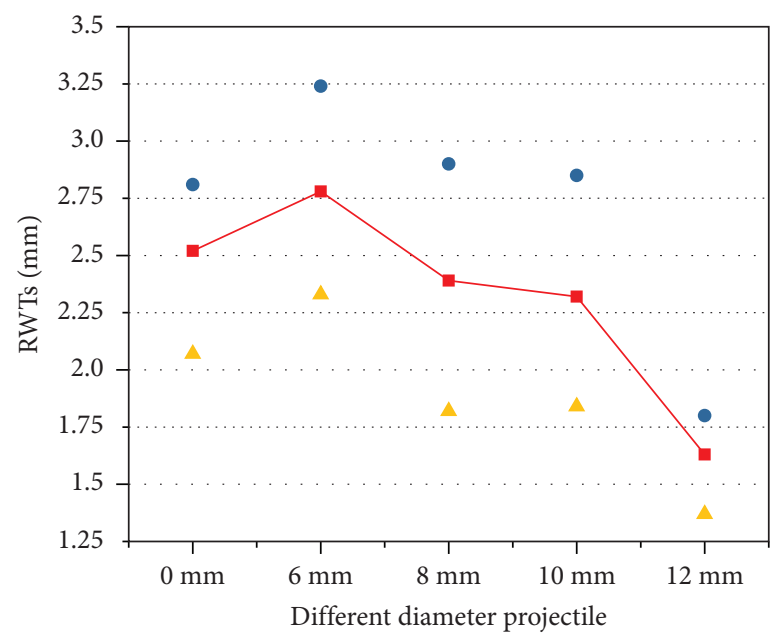

- RWTs

(c)

FIgUre 5: Comparison of RWTs between WAIM and different diameters projectile W-PAIM pipe: (a) transverse section of WIAM and W-PAIM pipes, (b) longitudinal section of WIAM and W-PAIM pipes, and (c) RWTs and error bar.

the graph, it can be concluded that the RWT of the pipes shaped by the W-PAIM process decreases with the increase in the diameter of the projectile.

\subsection{Penetration Behavior of the Projectile at Straight Segment.} In order to investigate the penetration behavior of the projectile at the straight segment, the velocity, pressure, and strain rates near the penetration front were explored and compared with those of WAIM.

Figure 6 shows the comparison of the velocity distribution near the penetration front at the middle location of the straight segment between WAIM and W-PAIM. For the convenience of observation, the penetration interfaces are overlay-displayed on the velocity magnitude contour, as shown in Figures 6(a) and 6(b). It can be seen for both processes the cross sections of the velocity field near the penetration front increase remarkably and the velocity fields in front of the penetration fronts are very uniform. The quantitative comparison of the velocity at three positions of $0.04,0.05$, and $0.06 \mathrm{~mm}$ from the entrance, marked by $\mathrm{x}-0.04$, $\mathrm{x}-0.05$, and $\mathrm{x}-0.06$, is shown in Figures 6(c)-6(e), respectively. Both the velocity profiles of WAIM and W-PAIM at $\mathrm{x}-0.04$, the position behind the penetration front, are approximately parabolic, which is a characteristic of pressure flow of Newtonian fluid. The velocities near the wall, or rather in the RWT zone, are almost zero. That means the RWTs would be almost unchanged after the penetration. Compared with the velocity profile of WAIM, the velocity profile of W-PAIM has a wider radial range and a smaller value at the center of cavity for its larger penetration section and the block of the rigid projectile. The velocity at the center in WAIM reaches $12 \mathrm{~m} / \mathrm{s}$ and that in W-PAIM is about $10 \mathrm{~m} / \mathrm{s}$. Both velocities are much faster than $0.34 \mathrm{~m} / \mathrm{s}$, a critical velocity of laminar flow and turbulence of water. Therefore, water flows in turbulent state. It shows the rationality of using the turbulent model for water in the simulation. At the current position of the penetration front, that is, $\mathrm{x}-0.05$, the velocity profile of $\mathrm{W}$-PAIM showed 


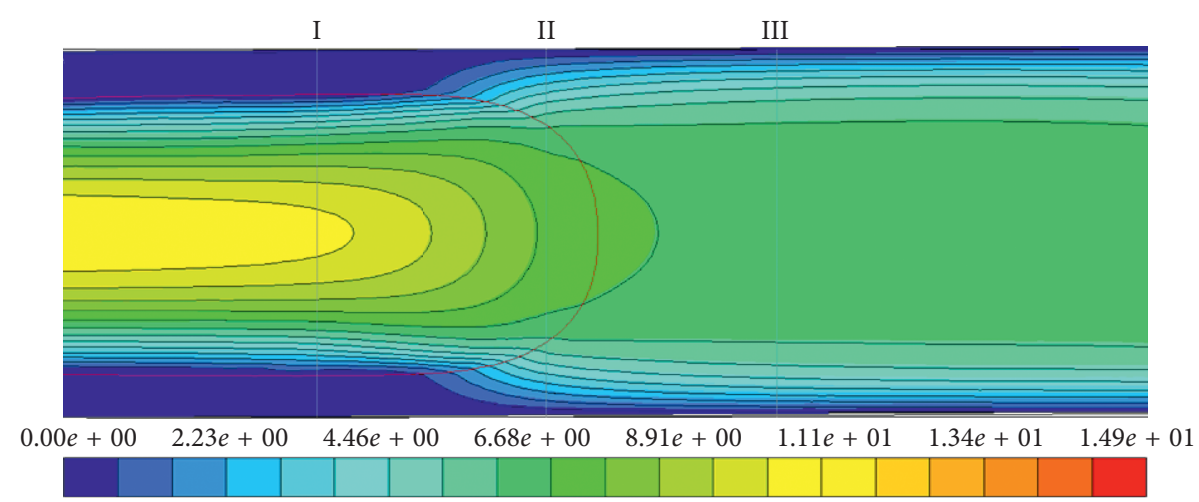

(a)

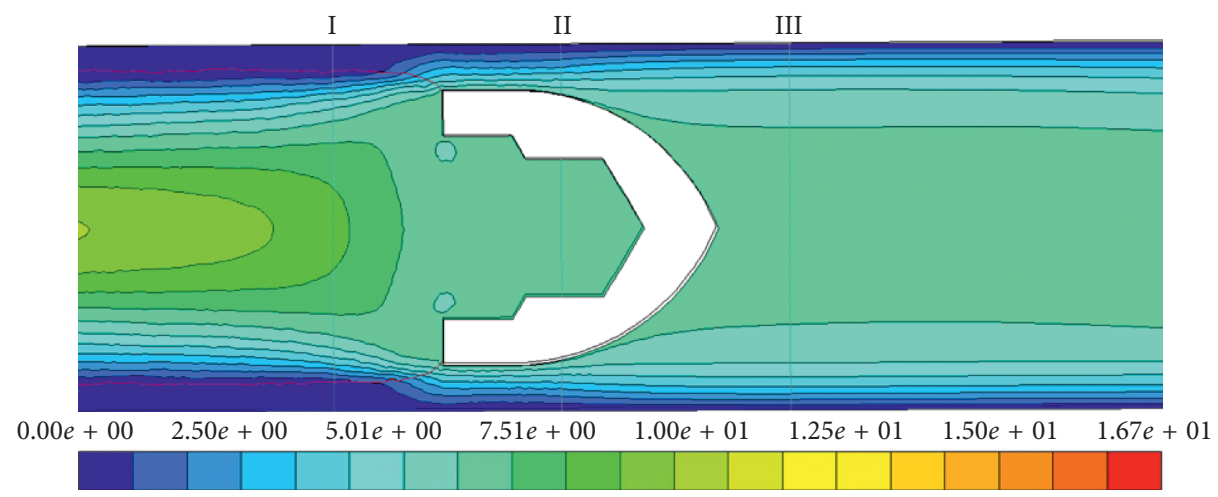

(b)

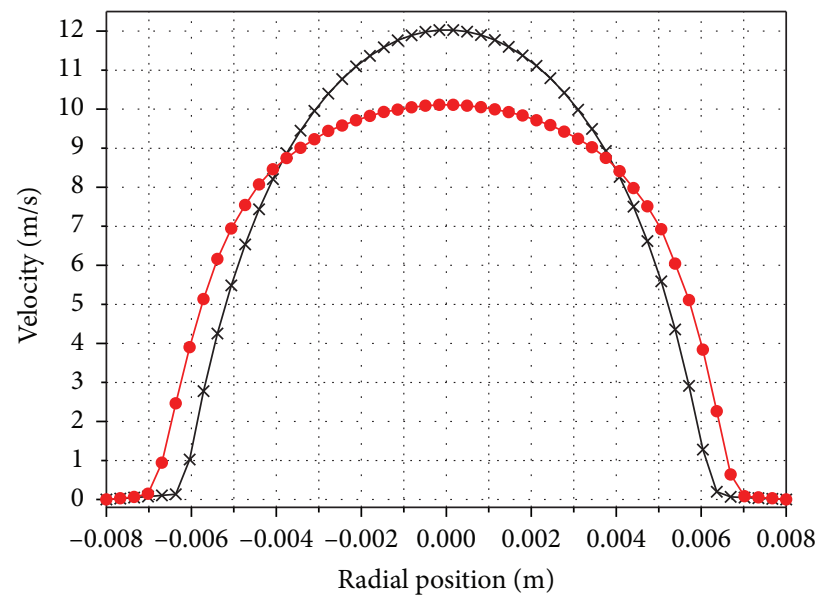

$*$ WAIM

$\rightarrow$ W-PAIM

(c)

Figure 6: Continued. 


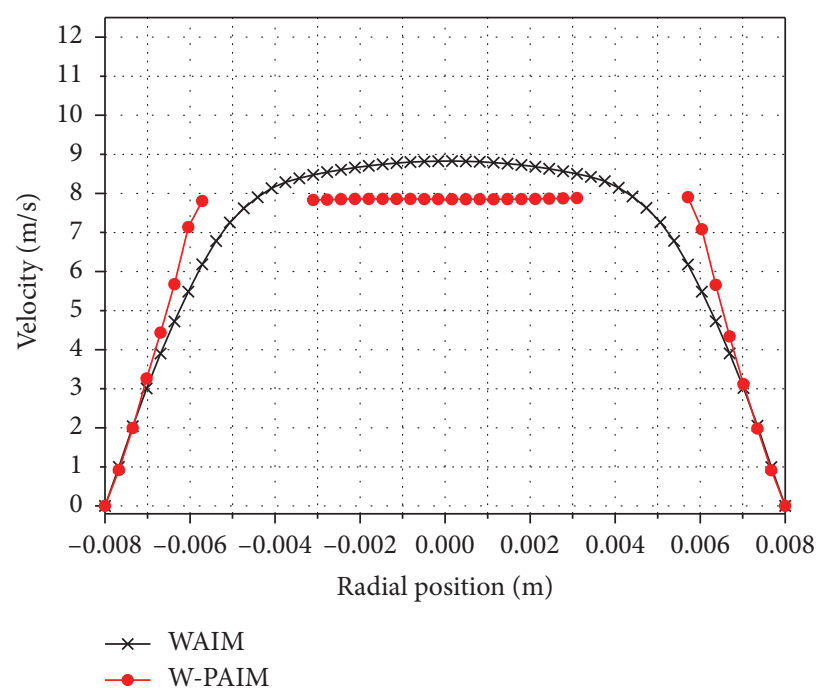

(d)

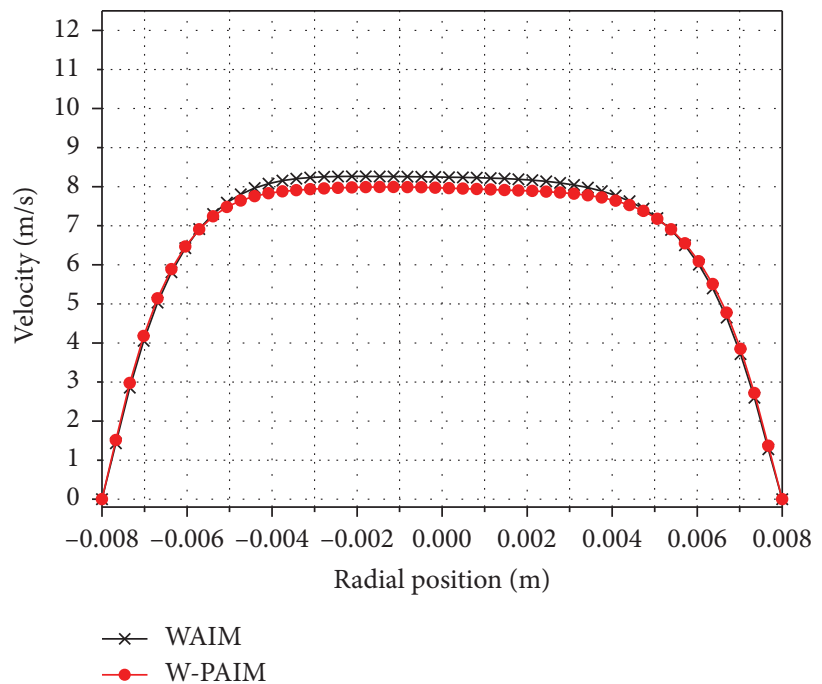

(e)

FIGURE 6: Comparison of the velocity distribution near the penetration front at the middle location of straight segments between WAIM and W-PAIM: (a) velocity magnitude contour of WAIM, (b) velocity magnitude contour of W-PAIM, (c) velocity profiles at position I, (d) velocity profiles at position II, (e) velocity profiles at position III (I denotes the location of $0.04 \mathrm{~mm}$, II is $0.05 \mathrm{~mm}$, and III is $0.06 \mathrm{~mm}$ ).

the shape of an isosceles trapezoid without velocity segments of near zero. The horizontal segment at the middle represented the velocity of the water trapped by the projectile. The linear segments on both sides were the velocity of the melt at the outside of the projectile, which implied that the melt flow at the outside of the projectile is drag flow. Moreover, the velocity of the drag flow at the outside of the projectile is faster than that of the subsequent pressure flow near the wall. Consequently, the water followed the projectile is partially supplemented to the drag flow area. Therefore, the simulated RWT is thinner than the radius difference between the part cavity and the projectile. Compared with the velocity field at $\mathrm{x}-0.04$, the cross sections of the velocity field at $\mathrm{x}-0.05$ increase remarkably and the velocity profile has relatively flat middle segments, which indicates the flow there is a plunger flow. The velocity at the center in WAIM is decreased and close to $9 \mathrm{~m} / \mathrm{s}$ and that in W-PAIM is about $8 \mathrm{~m} / \mathrm{s}$. The velocity profile of WAIM at $\mathrm{x}-0.06$, the position in front of the penetration front, is similar to that of W-PAIM. Both of them have flat middle segments, which indicate the flow before the penetration front is a typical plunger flow of pseudoplastic flow of the melt with a non-Newtonian index of 0.3 . The highest velocity in WAIM is further reduced to near $8.3 \mathrm{~m} / \mathrm{s}$ and that in W-PAIM basically keeps constant of about $8 \mathrm{~m} / \mathrm{s}$.

The pressure fields of WAIM and W-PAIM processes when the penetration front reaches the middle of the straight section are also compared, as shown in Figure 7. For both processes, the pressure behind the penetration front is the injection pressure due to almost no pressure loss in water. The pressure contours after a short distance to the penetration front in W-PAIM are similar to those in WAIM. The difference is that the pressure contours in front of the W-PAIM 


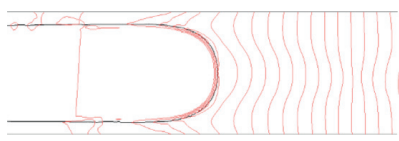

(a)

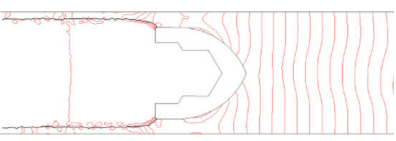

(b)

FIgURE 7: Comparison of the pressure contours near the penetration front at the middle location of straight segments between WAIM and W-PAIM: (a) WAIM and (b) W-PAIM.

projectile are straight lines for the existence of the rigid projectile, while the pressure contours in front of the WAIM penetration front are curves with shape like the penetration front.

Figure 8 shows the comparison of the strain rate distribution near the penetration front at the middle location of the straight segment between WAIM and W-PAIM. In order to observe conveniently, the penetration interfaces are overlay-displayed on the strain rate magnitude contour, as shown in Figures $8(\mathrm{a})$ and $8(\mathrm{~b})$. It can be seen for both processes that the strain rate peak behind the penetration front locates at the penetration interface, while that before the penetration front locates near the wall. The quantitative comparison of the velocity at three positions of $0.04,0.05$, and $0.06 \mathrm{~mm}$ from the entrance is shown in Figures 8(c)$8(\mathrm{e})$, respectively. At $\mathrm{x}-0.04$, the strain rate peak of W-PAIM is $5500 \mathrm{1} / \mathrm{s}$ and locates in the radius range of $0.0065 \mathrm{~mm}$, while that of WAIM is $5100 \mathrm{l} / \mathrm{s}$ and locates at a radial position of $0.006 \mathrm{~mm}$. Moreover, in WAIM, the strain rate closer to the center of cavity is lower,while the strain rate of W-PAIM is relatively uniform near to the center of cavity for the block of projectile. At $\mathrm{x}-0.05$, the current position of penetration front, the strain rate profiles are quite different between WAIM and W-PAIM. In WAIM, the strain rate peak locates near the wall, and the strain rate is relatively uniform in the radius range of 0.004 . While the strain rate peak in W-PAIM locates at the surface of projectile, and closer to the wall, the strain rate is lower. At the location of $\mathrm{x}-0.06$, the strain rate profile of W-PAIM is similar to that of WAIM. The strain rate peak locates close to the wall and is about $4500 \mathrm{~s}^{-1}$. The strain rate is almost zero in the radius range of 0.004 .

3.3. Penetration Behavior of the Projectile at Curved Segment. The penetration behavior of the projectile at the curved segment was explored by observing the velocity, pressure, and strain rates near the penetration front and compared with those of WAIM.

Figure 9 shows the comparison of the velocity distribution near the penetration front at the curved segment between WAIM and W-PAIM. For the convenience of investigation, the penetration interfaces are overlay-displayed on the velocity magnitude contour, as shown in Figures 9(a) and 9(b). It can be seen for both processes the cross sections of the velocity field near the penetration front increase remarkably and the velocity fields in front of the penetration fronts near the center of cavity are very uniform. The quantitative comparison of the velocity at position 4 , position 5 , and position 6 , marked by IV, V, and VI, is shown in
Figures 9(c)-9(e), respectively. The left side of the zero position is the outer convex side of the curved section and the right side is the inner concave side.

At the start position of the curved segment, as it can be seen from Figure 9(c) that both the velocity profiles of WAIM and W-PAIM are like parabolic and slightly skewed to the right, which is the inner concave side of the curved section. That indicates that the penetration tends to be close to the inner concave side at the start position of the curved segment. Compared with the velocity profile of WAIM, the velocity profile of W-PAIM has a wider range and a smaller value near the center of cavity. The velocity at the center in WAIM reaches $16 \mathrm{~m} / \mathrm{s}$ and that in W-PAIM is about $12 \mathrm{~m} / \mathrm{s}$.

At the current position of the penetration front, as it can be seen from Figure 9(d) that the projectile velocity close to the outer convex side in W-PAIM is above $12.5 \mathrm{~m} / \mathrm{s}$ and higher than that close to the inner concave side, which is about $8.5 \mathrm{~m} / \mathrm{s}$. Therefore, the projectile turns accordingly. However, the velocity profile of WAIM is still slightly skewed to the inner concave side with a maximum of about $13 \mathrm{~m} / \mathrm{s}$. The velocity profile of WAIM at VI, the position in front of the penetration front, is similar to that of W-PAIM. Both are skewed to the left, which is the outer convex side of the curved section. It can be seen from Figures $9(a)$ and $9(b)$ that the velocity contours with high value after the bending segment in both WAIM and W-PAIM processes deflect from the outer convex side to the center of cavity and tend to be symmetrical.

The pressure fields of WAIM and W-PAIM processes when the penetration reaches the middle of the curved section are also compared, as shown in Figure 10. The pressure contours near the penetration front in W-PAIM are similar to those in WAIM.

Figure 11 shows the comparison of the strain rate distribution near the penetration front at the curved segment between WAIM and W-PAIM. In order to observe conveniently, the penetration interfaces are overlay-displayed on the strain rate magnitude contour, as shown in Figures 11(a) and 11(b). It can be seen for both processes that the maximum strain rate behind the penetration front locates at the penetration interface, while that before the penetration front locates near the wall. The quantitative comparison of the velocity at three positions of IV, V, and VI is shown in Figures 11(c)-11(e), respectively. At position IV, the strain rate peak of W-PAIM near the outer convex side is about $5600 \mathrm{1} / \mathrm{s}$ and locates in the radius of $0.0055 \mathrm{~mm}$, and it is about $9500 \mathrm{1} / \mathrm{s}$ near the inner concave side and locates in the radius of $0.0065 \mathrm{~mm}$. That is to say, the strain rate on the outside is higher than that on the inside. However, the strain rate peak of WAIM near the outer convex side is about 10000 


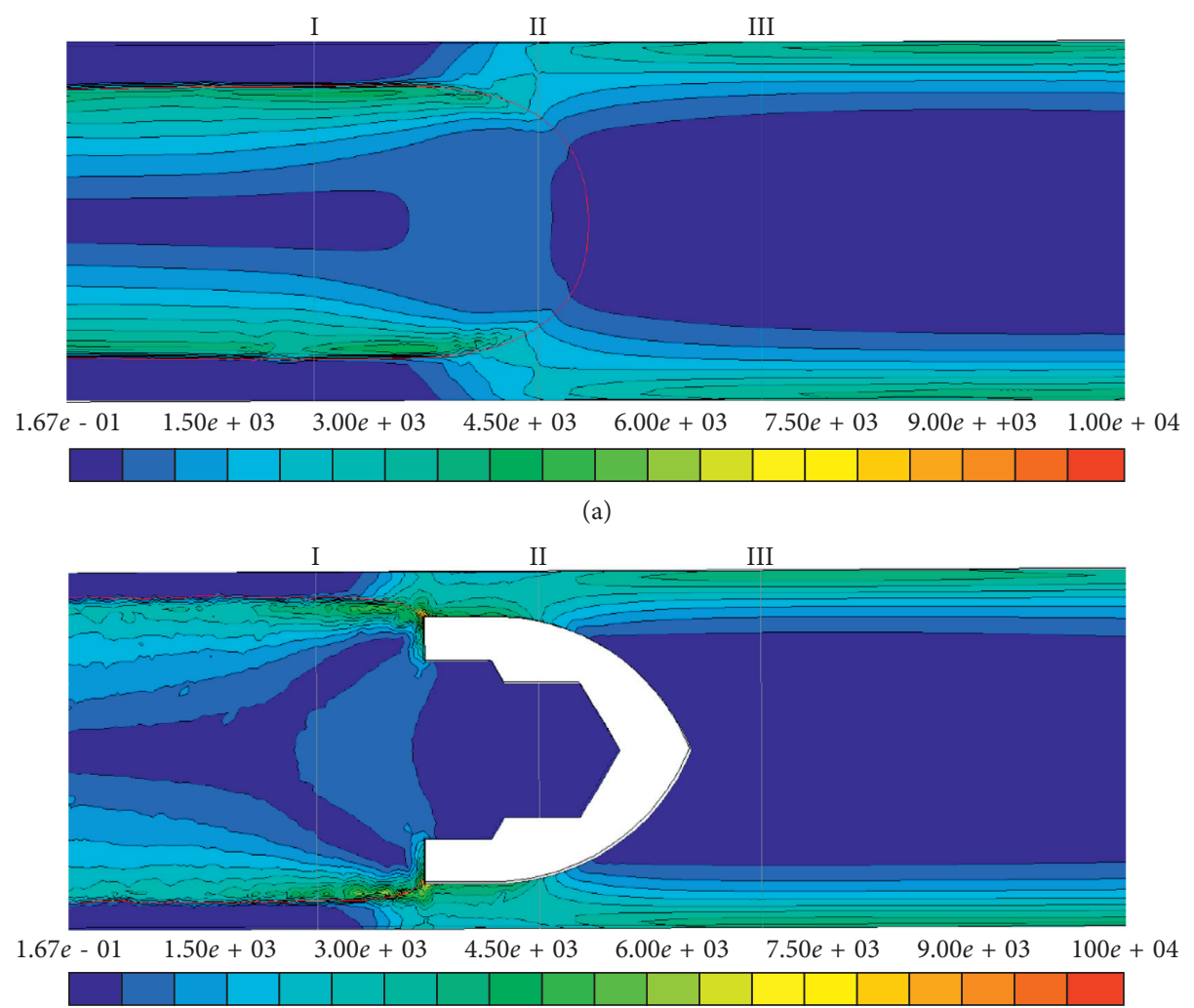

(b)

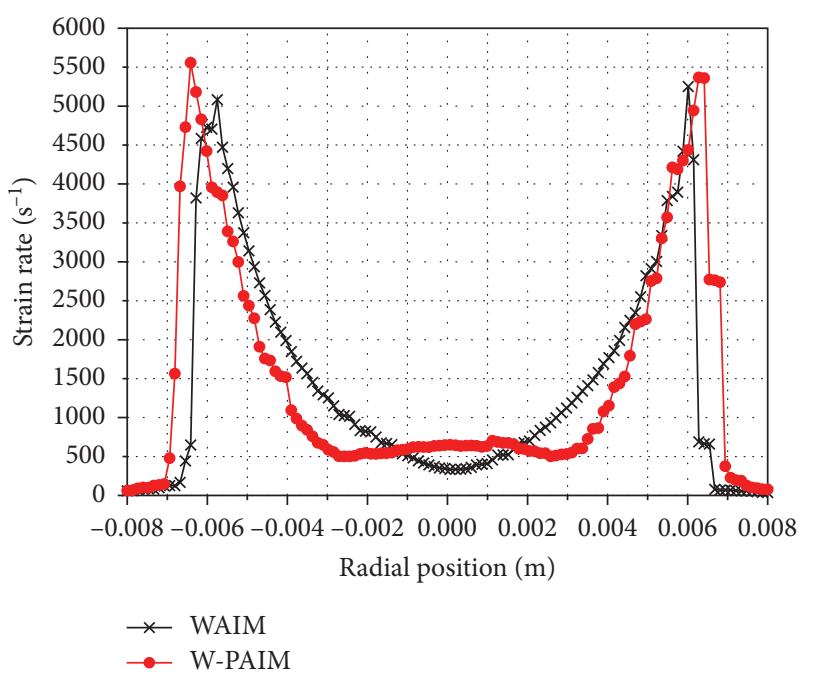

(c)

Figure 8: Continued. 


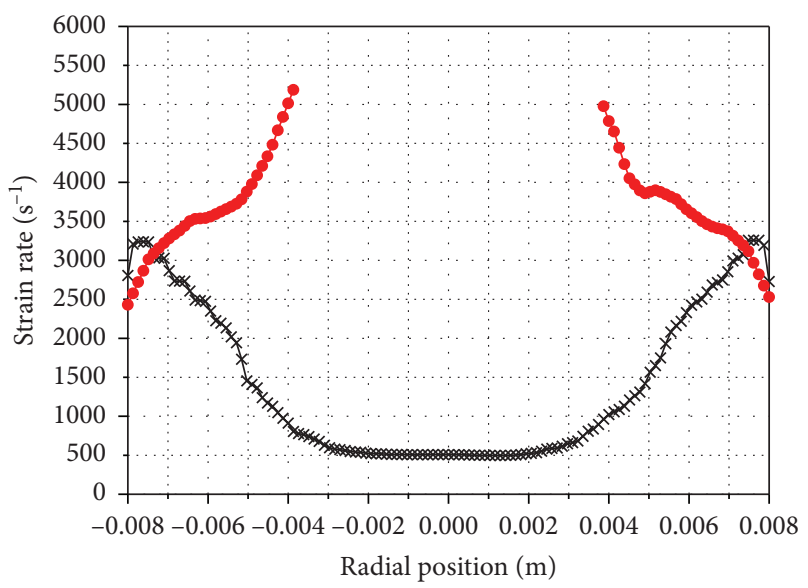

$\rightarrow$ WAIM

$\rightarrow$ W-PAIM

(d)

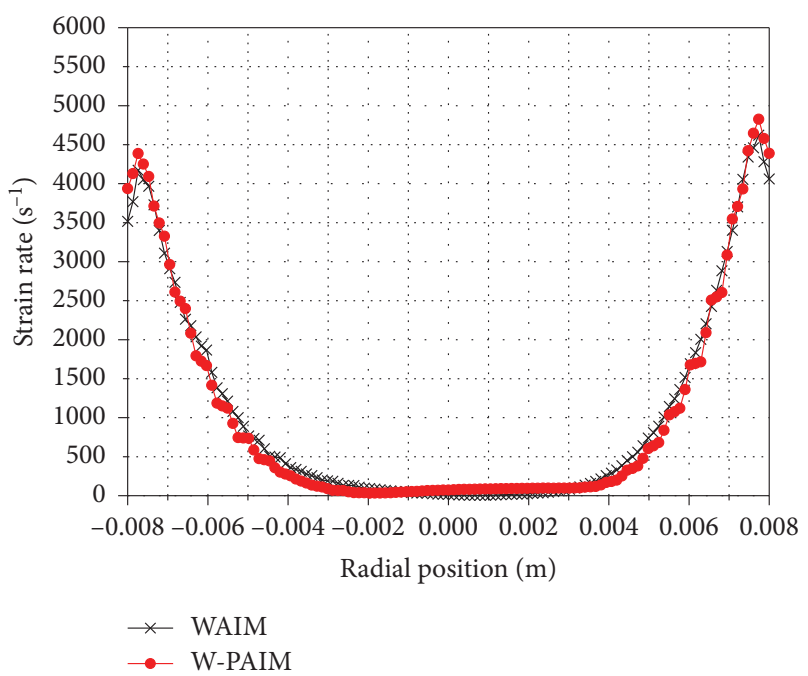

(e)

FIGURE 8: Comparison of the strain rate distribution near the penetration front at the middle location of straight segments between WAIM and W-PAIM: (a) strain rate magnitude contour of WAIM, (b) strain rate magnitude contour of W-PAIM, (c) strain rate profiles at position I, (d) strain rate profiles at position II, and (e) strain rate profiles at position III (I denotes the location of $0.04 \mathrm{~mm}$, II is $0.05 \mathrm{~mm}$, and III is $0.06 \mathrm{~mm})$.

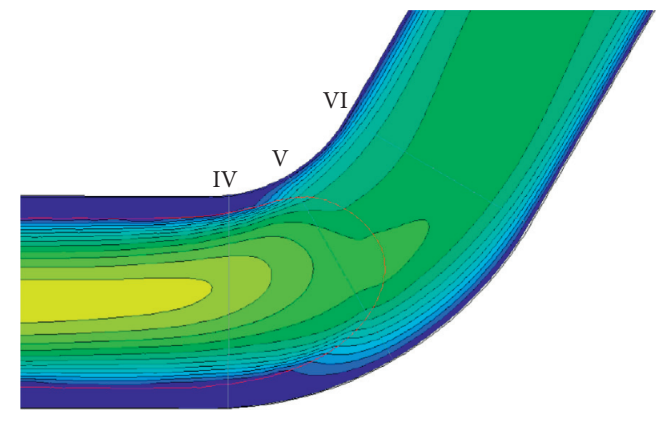

(a)
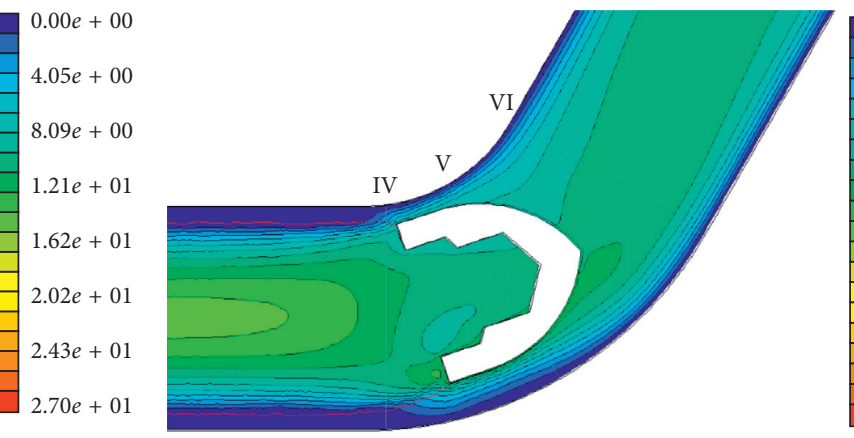

(b)

Figure 9: Continued. 


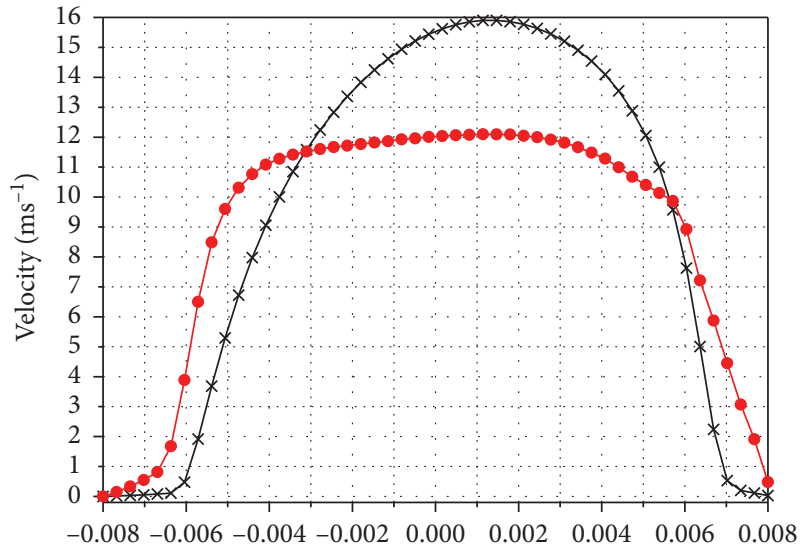

Radial position (m)

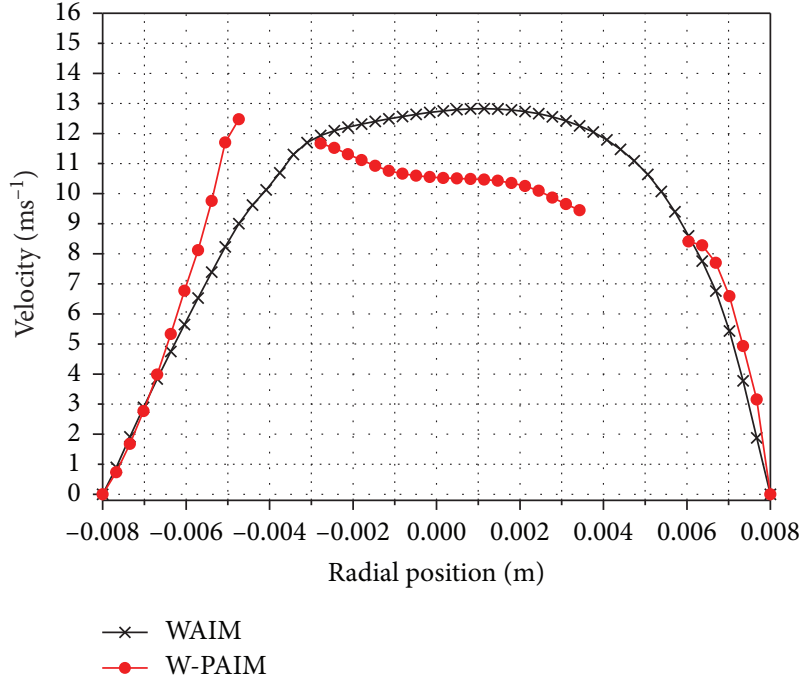

(d)

(c)

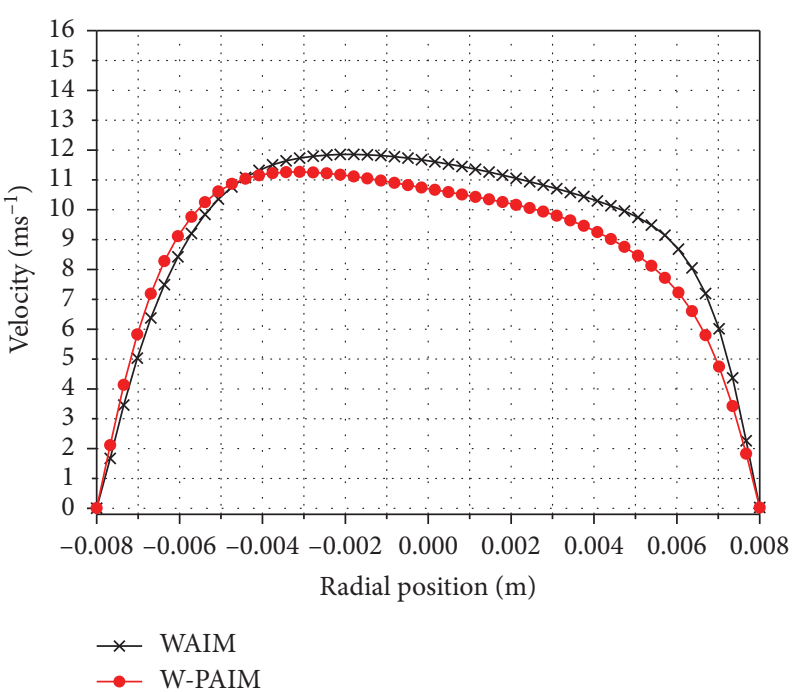

(e)

FIGURE 9: Comparison of the velocity distribution near the penetration front at the middle location of curved segments between WAIM and W-PAIM: (a) velocity magnitude contour of WAIM, (b) velocity magnitude contour of W-PAIM, (c) velocity profiles at position IV, (d) velocity profiles at position $\mathrm{V}$, and (e) velocity profiles at position VI (IV, V, and VI denote the beginning, middle, and end of the curved segments, respectively. Negative radial positions are near the outer convex side of curved segments, while the positive radial positions are near the inner concave side).

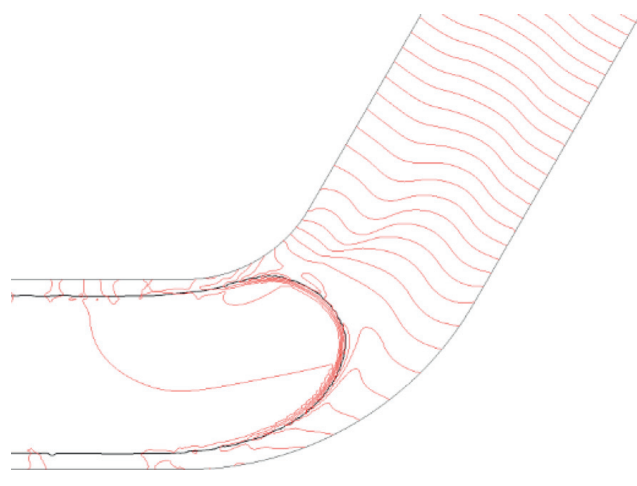

(a)

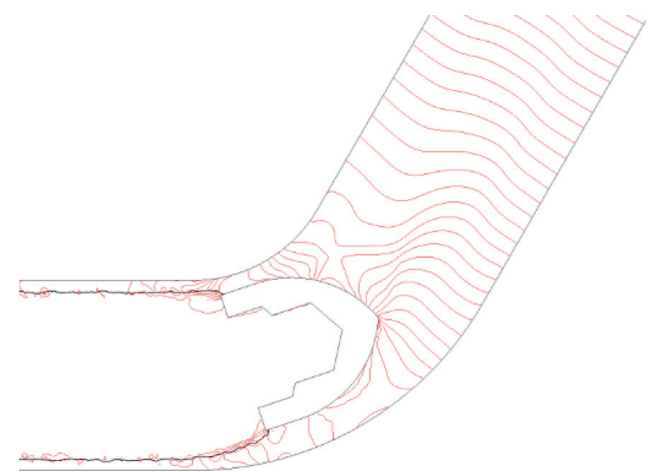

(b)

FIGURE 10: Comparison of the pressure contours near the penetration front at the middle location of curved segments between WAIM and W-PAIM: (a) WAIM and (b) W-PAIM. 


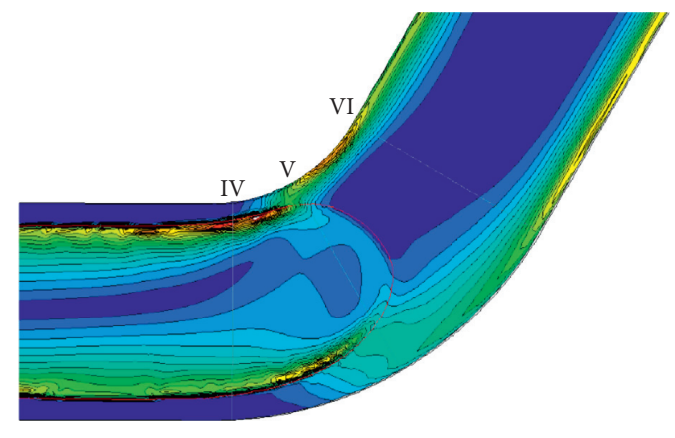

(a)

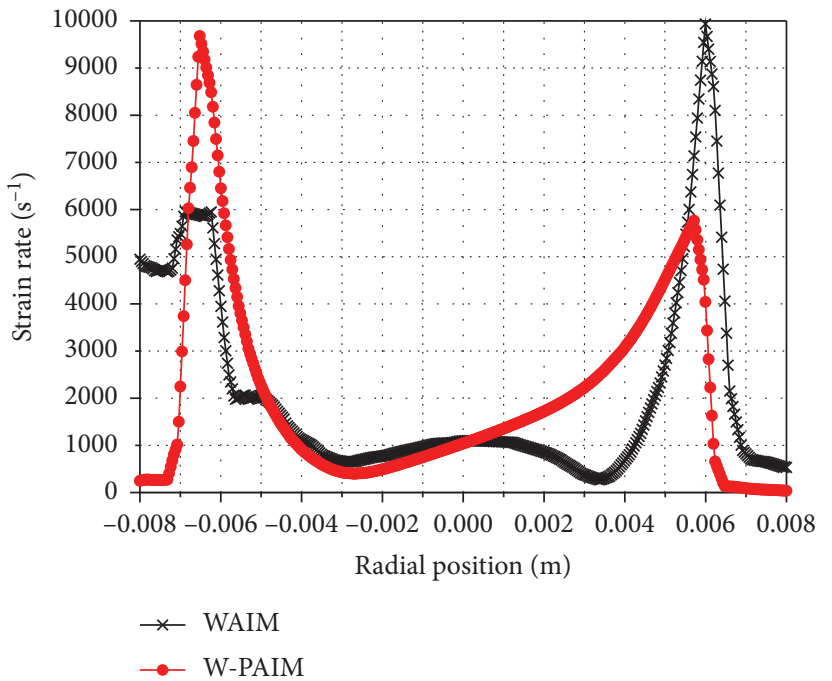

(c)

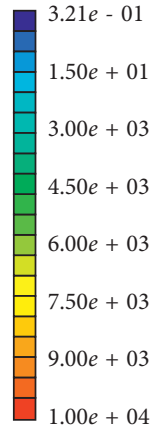

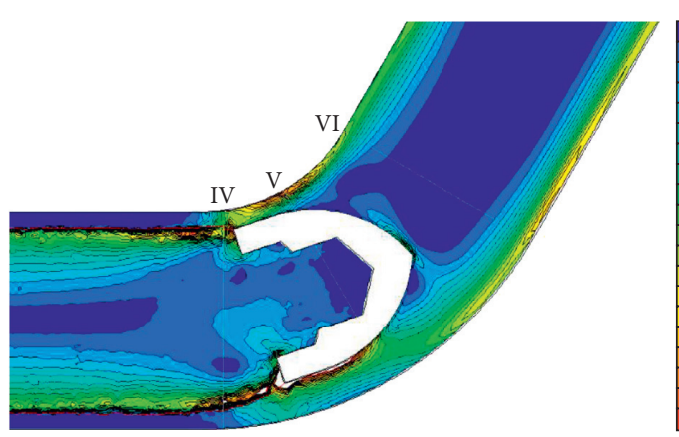

(b)

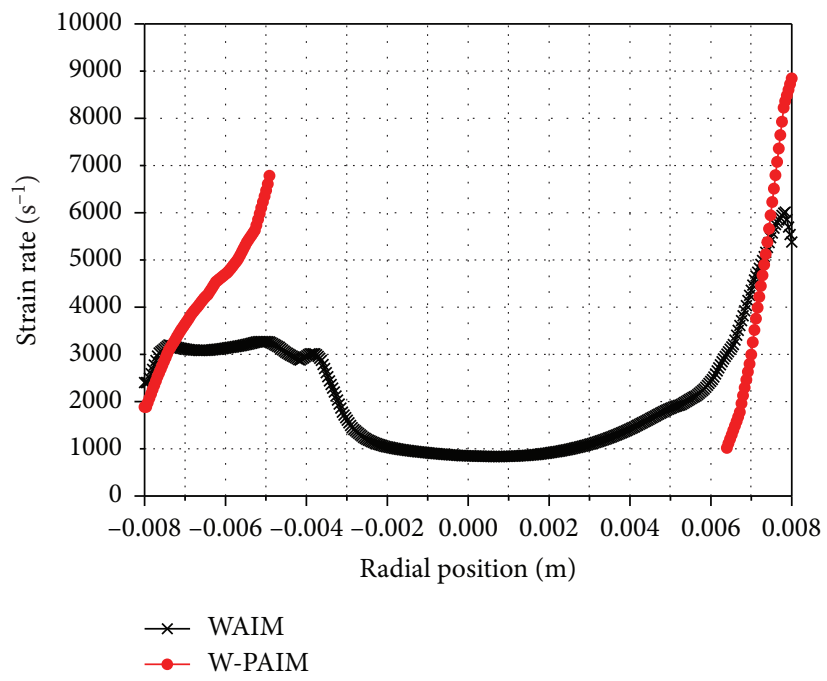

(d)

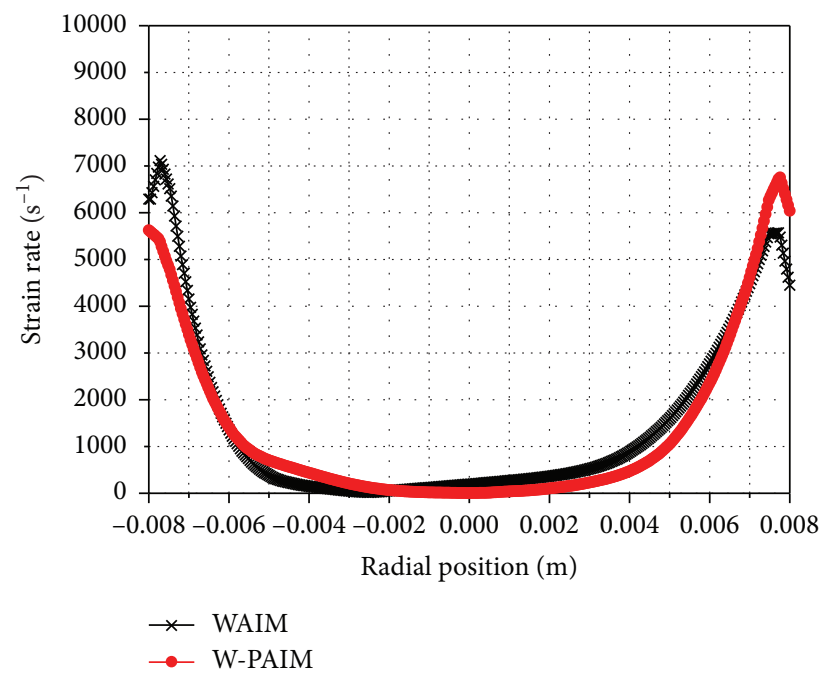

(e)

FIgURE 11: Comparison of the straight rate distribution near the penetration front at the middle location of curved segments between WAIM and W-PAIM: (a) straight rate magnitude contour of WAIM, (b) straight rate magnitude contour of W-PAIM, (c) velocity profiles at position IV, (d) straight rate profiles at position V, and (e) straight rate profiles at position VI (IV, V, and VI denote the beginning, middle, and end of the curved segments, respectively. Negative radial positions are near the outer convex side of curved segments, while the positive radial positions are near the inner concave side).

$1 / \mathrm{s}$ and locates in the radius of $0.006 \mathrm{~mm}$, and it is about $60001 / \mathrm{s}$ near the inner concave side and locates in the radius of $0.0065 \mathrm{~mm}$. At the start position of the curved section, the strain rate peak on the outside is about $40001 / \mathrm{s}$ higher than that on the inside in W-PAIM, while it is opposite in WAIM. At position $\mathrm{V}$, the current position of penetration front, both 
the strain rate peaks in WAIM and W-PAIM near the inner concave side are close to the wall. The strain rate peak in $\mathrm{W}$-PAIM near the outer convex side is close to the projectile, while that in WAIM is uniform. At position VI, the strain rate profile of W-PAIM is approach to that of WAIM with the peak close to the wall. The strain rate peak of W-PAIM near the outer convex side is about $5500 \mathrm{1} / \mathrm{s}$, and it is about $6700 \mathrm{l} / \mathrm{s}$ near the inner concave side. The strain rate peak of WAIM near the outer convex side is about $7100 \mathrm{l} / \mathrm{s}$, and it is about $5500 \mathrm{1} / \mathrm{s}$ near the inner concave side. In other words, at the end position of curved section, the strain rate peak on the outside is about $1000 \mathrm{1} / \mathrm{s}$ less than that on the inside in W-PAIM, while it is opposite in WAIM.

\section{Conclusion}

According to the simulation results of the water injection stage of a W-PAIM pipe with straight and curved segments and the comparison with those of WAIM pipe, the following conclusions can be drawn:

(1) When the diameter of the projectile is $12 \mathrm{~mm}$, the RWTs of the W-PAIM pipe are much thinner than those of the WAIM pipe in the case studied. In addition, at the curved segments, the RWTs are thinner at the inner concave side and thicker at the outer convex side. The projectile has a crucial influence on the RWT. The penetrations of the projectile in W-PAIM are relatively stable at the straight segment and always close to the inner concave side. Moreover, the uniform RWT near the end of part cavity can be obtained by designing a slender rather than a bulky overflow cavity for a constant pressure gradient. When the diameter of the projectile is less than $10 \mathrm{~mm}$, there is a certain deflection phenomenon during projectile passing the melt and the RWT fluctuates greatly. The RWT of the pipes shaped by the W-PAIM process decreases with the increase in the diameter of the projectile.

(2) At the straight segment, both the velocity profiles of WAIM and W-PAIM at the position behind the penetration front are approximately parabolic for the pressure flow of Newtonian fluid there. The velocities in the RWT zone are almost zero. Compared with the velocity profile of WAIM, the velocity profile of W-PAIM has a wider radial range for the larger penetration section and a smaller value at the center of cavity for the block of the rigid projectile. At the position of the penetration front, the velocity profile of W-PAIM showed the shape of an isosceles trapezoid, which implies the melt flow at the outside of the projectile is drag flow. The velocity profile of W-PAIM at the position in front of the penetration front is approach to that of WAIM. Both have characteristics of a typical plunger flow of pseudoplastic flow of the melt with a non-Newtonian index of 0.3.

(3) At the curved segment, both the velocity profiles of WAIM and W-PAIM at the position behind the penetration front are like parabolic and slightly skewed to the inner concave side. That indicates the penetration tends to be close to the inner concave side. At the position of the penetration front, the projectile velocity close to the outer convex side in W-PAIM is higher than that close to the inner concave side, and the projectile's turning is realized. While the velocity profile of WAIM is still slightly skewed to the inner concave side. The velocity profile of W-PAIM at the position in front of the penetration front is similar to that of WAIM and both are skewed to the outer convex side and higher at that side. The velocity contours at the straight segment after the curved segment in both WAIM and W-PAIM processes deflect from the outer convex side to the center of cavity and tend to be symmetrical again.

(4) The pressure fields of W-PAIM and WAIM are very similar in both straight and curved segments. The existence of the projectile only affects the pressure field in a very small region around its location.

(5) In both W-PAIM and WAIM, the strain rate peak behind the penetration front locates at the penetration interface, while that in front of the penetration front locates near the wall. At the penetration front in straight segment, the strain rate peak of WAIM locates near the wall, while that of W-PAIM locates near the projectile outer surface. At the penetration front in the curved segment, the strain rate peak near the inner concave side in both WAIM and W-PAIM locates near the wall, while that near the outer convex side in $\mathrm{W}$-PAIM locates near the penetration interface.

\section{Data Availability}

The data used to support the findings of this study are available from the corresponding author upon request.

\section{Conflicts of Interest}

The authors declare that there are no conflicts of interest regarding the publication of this paper.

\section{Acknowledgments}

This study was supported financially by the National Natural Science Foundation of China (NSFC) (nos. 51563010 and 21664002) for which the authors are very grateful. And this work was also supported financially by the Natural Science Foundation of China of Jiangxi Province (no. 20181BAB206014) for which the authors are grateful.

\section{References}

[1] M. R. Kamal, A. I. Isayev, and S. J. Liu, Eds., Injection Molding: Technology and Fundamentals, Hanser Gardner Publications, Cincinnati, OH, USA, 2009.

[2] S. J. Liu, "Water assisted injection molding: a review," International Polymer Processing, vol. 24, no. 2, pp. 315-325, 2009.

[3] W. Michaeli, A. Brunswick, and T. Pohl, "A comparison of gas and water," Kunststoffe-Plast Europe, vol. 89, no. 9, pp. 56-60, 1999. 
[4] S. Sannen, J. De Keyzer, and P. Van Puyvelde, "The influence of melt and process parameters on the quality and occurrence of part defects in water-assisted injection molded tubes," International Polymer Processing, vol. 26, no. 5, pp. 551-559, 2011.

[5] S. Sannen, M. De Munck, P. Van Puyvelde, and J. De Keyzer, "Water penetration behavior in water-assisted injection molding (WAIM): a study of product quality for different process and material parameters," International Polymer Processing, vol. 27, no. 5, pp. 602-616, 2012.

[6] IIDA and ISAO, "Manufacture of hollow body," IIDA, ISAO, Waterford, Ireland, JPH04208425, 1992.

[7] H. Eckardt, "Making parts lightweight strong with new technologies," Plastics Technology, vol. 52, no. 2, 2012.

[8] S.-J. Liu and Y.-C. Wu, "Dynamic visualization of cavityfilling process in fluid-assisted injection molding-gas versus water," Polymer Testing, vol. 26, no. 2, pp. 232-242, 2007.

[9] A. Polynkin, L. Bai, J. F. T. Pittman et al., "Water assisted injection moulding: development of insights and predictive capabilities through experiments on instrumented process in parallel with computer simulations," Plastics, Rubber and Composites, vol. 37, no. 2-4, pp. 131-141, 2008.

[10] T. Kuang, C. Yu, and Y. Deng, "Influence of cross section of cavity on water penetration during overflow water-assisted injection molding process," Journal of Chemical Industry and Engineering, vol. 65, no. 10, pp. 4176-4182, 2014.

[11] Y. Chen, H. Zhou, Z. Zhang, and H. Kong, "Comparison research on residual wall thickness of water-assisted and gasassisted injection molded parts," China Plastics, vol. 26, no. 6, pp. 71-75, 2012.

[12] H.-X. Huang and Z.-W. Deng, "Effects and optimization of processing parameters in water-assisted injection molding," Journal of Applied Polymer Science, vol. 108, no. 1, pp. 228235, 2008.

[13] J. Yang, X. Zhou, and G. Luo, "Study of water penetration length and processing parameters optimization in waterassisted injection molding," The International Journal of Advanced Manufacturing Technology, vol. 69, no. 9-12, pp. 2605-2612, 2013.

[14] J. Yang, X. Zhou, and Q. Niu, "Model and simulation of water penetration in water-assisted injection molding," The International Journal of Advanced Manufacturing Technology, vol. 67, no. 1-4, pp. 367-375, 2013.

[15] C. Hopmann, W. Michaeli, M. Gründler, O. Grönlund, and A. Neuss, "Process development of the projectile injection technique (PIT)," in Proceedings of the Annual Technical Conference of the Society of Plastics Engineers 2011, (ANTEC 2011), pp. 1706-1710, Boston, MA, USA, May 2011.

[16] C. Hopmann and C. Behmenburg, "Hollow bodies using the projectile injection technique," in Proceedings of the Annual Technical Conference of the Society of Plastics Engineers 2013, (ANTEC 2013), pp. 282-285, Cincinnati, OH, USA, April 2013.

[17] C. Hopmann and U. Recht, "Manufacturing of fibre-reinforced, elastomeric parts using the injection molding process," in Proceedings of the Annual Technical Conference of the Society of Plastics Engineers 2015, (ANTEC 2015), pp. 17281732, Orlando, FL, USA, March 2015.

[18] T. Kuang, J. Pan, Q. Feng et al., "Residual wall thickness of water-powered projectile-assisted injection molding pipes," Polymer Engineering \& Science, vol. 59, no. 2, pp. 295-303, 2019.

[19] W. Liu, T. Kuang, and D. Lai, "Preliminary experimental investigation on the fluid-projectile-assisted injection molding pipes," China Plastics Industry, vol. 44, no. 11, pp. 138-142, 2016. 